\title{
Lie expression for multi-parameter Klyachko idempotent
}

\section{Askar Dzhumadil'daev}

Received: 15 September 2010 / Accepted: 17 September 2010 / Published online: 9 October 2010

(C) Springer Science+Business Media, LLC 2010

\begin{abstract}
An expression for the multi-parameter Klyachko idempotent as a linear combination of Lie base elements is given.
\end{abstract}

Keywords Free Lie algebras · Lie elements · Klyachko idempotent

\section{Introduction}

Let $\mathcal{F}$ be the free associative algebra with generators $a_{1}, a_{2}, \ldots$, over the field of complex numbers $\mathbf{C}$. Its elements are associative polynomials in non-commuting variables $a_{1}, a_{2}, \ldots$, with coefficients from $\mathbf{C}$. Let $\mathcal{F}^{-}$be the Lie algebra of $\mathcal{F}$ under commutator $[a, b]=a b-b a$. Endow $\mathcal{F}$ with the structure of a Hopf algebra by defining a coproduct $\delta$ such that $\delta\left(a_{i}\right)=a_{i} \otimes 1+1 \otimes a_{i}$. Let $\mathcal{L}$ be the Lie subalgebra of $\mathcal{F}$ generated by $a_{1}, a_{2}, \ldots$ So $\mathcal{L}$ is the free Lie algebra on generators $a_{1}, a_{2}, \ldots$

An element $X=f\left(a_{1}, \ldots, a_{n}\right) \in \mathcal{F}$ is called a Lie element if $X \in \mathcal{L}$. There is a well-known criterion for $X$ to be a Lie element (Friedrich's criterion): $X$ is a Lie element if and only if $X$ is primitive element of $\mathcal{F}$ (considered as a Hopf algebra),

$$
\delta(X)=X \otimes 1+1 \otimes X .
$$

If $X$ is a homogeneous Lie element of degree $m$, the Dynkin-Specht-Wever theorem allows us to construct its Lie expression

$$
\pi X=m X
$$

where $\pi X$ denotes Lie element corresponding to $X$ constructed by

$$
\pi a_{i}=a_{i}, \quad \pi\left(a_{i_{1}} \ldots a_{i_{m}}\right)=\left[a_{i_{1}},\left[\cdots\left[a_{i_{m-1}}, a_{i_{m}}\right] \cdots\right]\right] .
$$

A. Dzhumadil'daev ( $\varangle)$

Kazakh-British University, Tole bi 59, Almaty, 050000, Kazakhstan

e-mail: dzhuma@hotmail.com 
See [4] or [9] for details. For example, $a_{1}^{2}$ is not Lie element, but $X=-a_{1}^{2} a_{2}+$ $2 a_{1} a_{2} a_{1}-a_{2} a_{1} a_{1}$ is a Lie element with $X=-\left[a_{1},\left[a_{1}, a_{2}\right]\right]$.

A.A. Klyachko in [5] has constructed the following idempotent in the group algebra of the permutation group:

$$
k_{n}=\frac{1}{n} \sum_{\sigma \in S_{n}} q^{m a j(\sigma)} \sigma .
$$

Recall that the major-index $\operatorname{maj}(\sigma)$ is defined as a sum of descent indices,

$$
\operatorname{maj}(\sigma)=\sum_{1 \leq i<n, \sigma(i)>\sigma(i+1)} i .
$$

An important property of the Klyachko idempotent is that the element

$$
k_{n}\left(a_{1}, \ldots, a_{n}\right)=\frac{1}{n} \sum_{\sigma} q^{m a j(\sigma)} a_{\sigma(1)} \cdots a_{\sigma(n)}
$$

is a Lie element, assuming $q$ is a primitive $n$-th root of unity. For example,

$$
2 k_{2}\left(a_{1}, a_{2}\right)=a_{1} a_{2}-a_{2} a_{1}=\left[a_{1}, a_{2}\right] \in \mathcal{L} .
$$

The case $n=3$ is not so evident. If

$$
q=-\frac{1}{2} \pm i \frac{\sqrt{3}}{2}
$$

then

$3 k_{3}\left(a_{1}, a_{2}, a_{3}\right)=a_{1} a_{2} a_{3}+q^{2} a_{1} a_{3} a_{2}+q a_{2} a_{1} a_{3}+q^{2} a_{2} a_{3} a_{1}+q a_{3} a_{1} a_{2}+q^{3} a_{3} a_{2} a_{1}$.

By Friedrich's criterion

$$
k_{3}\left(a_{1}, a_{2}, a_{3}\right) \in \mathcal{L},
$$

and by the Dynkin-Specht-Weber theorem,

$$
\begin{aligned}
& 3 k_{3}\left(a_{1}, a_{2}, a_{3}\right) \\
& =\left[\left[a_{1}, a_{2}\right], a_{3}\right]+q^{2}\left[\left[a_{1}, a_{3}\right], a_{2}\right]+q\left[\left[a_{2}, a_{1}\right], a_{3}\right]+q^{2}\left[\left[a_{2}, a_{3}\right], a_{1}\right] \\
& \quad+q\left[\left[a_{3}, a_{1}\right], a_{2}\right]+q^{3}\left[\left[a_{3}, a_{2}\right], a_{1}\right] .
\end{aligned}
$$

But one can check that the following is a simpler expression for $k_{3}$ as a Lie element,

$$
k_{3}\left(a_{1}, a_{2}, a_{3}\right)=\left[a_{1},\left[a_{2}, a_{3}\right]\right]+q\left[a_{2},\left[a_{1}, a_{3}\right]\right] \in \mathcal{L},
$$

where in all summands the last element $a_{3}$ seats in the last place.

In this paper we show that this kind of construction exists for any $n$. We give expression of the Klyachko element as a Lie element in a more general context. Let $[n]=\{1,2, \ldots, n\}$ and $S_{n}$ be its permutation group. We will use one-line notation 
for permutations: instead of $\left(\begin{array}{cccc}1 & 2 & \cdots & n \\ \sigma(1) & \sigma(2) & \cdots & \sigma(n)\end{array}\right)$ we will write $\sigma(1) \cdots \sigma(n)$. More generally we will consider words in the alphabet $[n]$ that have at most one occurrence of each letter $i \in[n]$. If every element of $[n]$ appears in such a word $u=i_{1} \ldots i_{k}$, then $k=n$ and we can consider $w$ as a permutation. For a word $u=i_{1} \ldots i_{k}$, say that $k=|u|$ is its length and that $s \in[n-1]$ is a descent index if $i_{s}>i_{s+1}$. Denote by $\operatorname{Des}(u)$ a set of descent indices of $u$. The sum of all descent indices, as we mentioned above, is called major index of $u$ and is denoted $\operatorname{maj}(u)$,

$$
\operatorname{maj}(u)=\sum_{j \in \operatorname{Des}(u)} j .
$$

Define the multi-parametric $\mathbf{q}$-major index $\operatorname{maj}_{\mathbf{q}}(u)$ of word $u$ by

$$
\operatorname{maj}_{\mathbf{q}}(u)=\frac{\prod_{j \in \operatorname{Des}(u)} q_{u(1)} \cdots q_{u(j)}}{\prod_{i=1}^{|u|-1}\left(1-q_{u(1)} \cdots q_{u(i)}\right)},
$$

where $q_{1}, \ldots, q_{n}$ are some variables.

Let $u=i_{1} \cdots i_{k}$ and $i_{s}=\max \left\{i_{1}, \ldots, i_{k}\right\}$ be maximum of the letters of $u$. Call the subwords $L(u)=i_{1} \ldots i_{s-1}$ the left part and $R(u)=i_{s+1} \cdots i_{k}$ the right part of $u$. For a word $u=i_{1} \cdots i_{k-1} i_{k}$ denote by $\operatorname{rev}(u)=i_{k} i_{k-1} \cdots i_{1}$ the word $u$ written in reverse order. For example, if $u=513942$, then

$$
\begin{gathered}
|u|=6, \quad \operatorname{Des}(u)=\{1,4,5\}, \quad \operatorname{maj}(u)=1+4+5=10, \\
\operatorname{maj}_{\mathbf{q}}(u)=\frac{q_{1}^{2} q_{3}^{2} q_{4} q_{5}^{3} q_{9}^{2}}{\left(1-q_{5}\right)\left(1-q_{1} q_{5}\right)\left(1-q_{1} q_{3} q_{5}\right)\left(1-q_{1} q_{3} q_{5} q_{9}\right)\left(1-q_{1} q_{3} q_{4} q_{5} q_{9}\right)}, \\
L(u)=513, \quad R(u)=42, \quad \operatorname{rev}(u)=249315 .
\end{gathered}
$$

Let $K(\mathbf{q})$ be the field of rational functions in $\mathbf{q}$ over the field $K$ and $K(\mathbf{q}) S_{n}$ be group algebra of symmetric group with coefficients in $K(\mathbf{q})$. There is a natural left action of $S_{n}$ on $K(\mathbf{q})$ : if $f(\mathbf{q})=f\left(q_{1}, \ldots, q_{n}\right) \in K(\mathbf{q})$, then we define

$$
\sigma[f(\mathbf{q})]=f\left(q_{\sigma(1)}, \ldots, q_{\sigma(n)}\right) .
$$

The "correct" product for $K(\mathbf{q}) S_{n}$ is not the straightforward analogue of the product for $K S_{n}$. Instead we need the twisted product of $f(\mathbf{q}) \sigma$ and $g(\mathbf{q}) \tau$, denoted $f(\mathbf{q}) \sigma \ltimes$ $g(\mathbf{q}) \tau$, defined by

$$
f(\mathbf{q}) \sigma \ltimes g(\mathbf{q}) \tau=(f(\mathbf{q}) \sigma[g(\mathbf{q})] \sigma \tau .
$$

For example,

$$
213 \ltimes \frac{q_{2} q_{3}}{\left(1-q_{2}\right)\left(1-q_{2} q_{3}\right)} 231=\frac{q_{1} q_{3}}{\left(1-q_{1}\right)\left(1-q_{1} q_{3}\right)} 132 .
$$

We recall the following multi-parameter generalization of Klyachko idempotent introduced in [7]. Namely, an element $k_{n}(\mathbf{q}) \in K(\mathbf{q}) S_{n}$ defined by

$$
k_{n}(\mathbf{q})=\sum_{\sigma \in S_{n}} \operatorname{maj}_{\mathbf{q}}(\sigma) \sigma
$$


is an idempotent in $K(\mathbf{q}) S_{n}$ under multiplication $\ltimes$. Moreover, $k_{n}(\mathbf{q})\left(a_{1}, \ldots, a_{n}\right)$ is a Lie element, if $q_{1} q_{2} \cdots q_{n}=1$, but $q_{i_{1}} q_{i_{2}} \cdots q_{i_{r}} \neq 1$, for any proper subset $\left\{i_{1}, \ldots, i_{r}\right\} \subset[n]$.

The multilinear part of the free Lie algebra of degree $n$ is $(n-1)$ !-dimensional and any multilinear Lie element should be a linear combination of base elements of a form $\left[a_{\sigma(1)},\left[\cdots\left[a_{\sigma(n-1)}, a_{n}\right] \cdots\right]\right]$. The existence of a Lie expression for the Klyachko element as such a linear combination was established in many papers (see for example [1-3, 8]).

In our paper we give an explicit such expression of the multi-parameter Klyachko element as a Lie word.

Theorem 1 If $q_{1} q_{2} \cdots q_{n}=1$, but $q_{i_{1}} q_{i_{2}} \cdots q_{i_{r}} \neq 1$, for any proper subset $\left\{i_{1}, \ldots, i_{r}\right\}$ of $\{1,2, \ldots, n\}$, then

$$
k_{n}(\mathbf{q})=\sum_{\sigma \in S_{n}, \sigma(n)=n} \operatorname{maj}_{\mathbf{q}}(\sigma)[\sigma(1),[\sigma(2), \cdots,[\sigma(n-1), n] \cdots]] .
$$

Equivalently,

$$
\sum_{\sigma \in S_{n}} m a j_{\mathbf{q}}(\sigma) a_{\sigma(1)} \cdots a_{\sigma(n)}=\sum_{\tau \in S_{n-1}} \frac{m a j_{\mathbf{q}}(\tau)\left[a_{\tau(1)},\left[a_{\tau(2)}, \cdots\left[a_{\tau(n-1)}, a_{n}\right] \cdots\right]\right]}{\left(1-q_{1} \cdots q_{n-1}\right)} .
$$

Example Let us demonstrate calculations for the case $n=3$. Denote by $H$ the righthand side of equality (1). We have

$$
\begin{array}{ll}
\operatorname{maj}_{\mathbf{q}}(123)=\frac{1}{\left(1-q_{1}\right)\left(1-q_{1} q_{2}\right)}, & \operatorname{maj}_{\mathbf{q}}(132)=\frac{q_{1} q_{3}}{\left(1-q_{1}\right)\left(1-q_{1} q_{3}\right)}, \\
\operatorname{maj}_{\mathbf{q}}(213)=\frac{q_{2}}{\left(1-q_{2}\right)\left(1-q_{1} q_{2}\right)}, & \operatorname{maj}_{\mathbf{q}}(231)=\frac{q_{2} q_{3}}{\left(1-q_{2}\right)\left(1-q_{2} q_{3}\right)}, \\
\operatorname{maj}_{\mathbf{q}}(312)=\frac{q_{3}}{\left(1-q_{3}\right)\left(1-q_{1} q_{3}\right)}, & \operatorname{maj}_{\mathbf{q}}(321)=\frac{q_{2} q_{3}^{2}}{\left(1-q_{3}\right)\left(1-q_{2} q_{3}\right)},
\end{array}
$$

and

$$
\begin{aligned}
k_{3}(\mathbf{q})(a, b, c)= & \frac{a b c}{\left(1-q_{1}\right)\left(1-q_{1} q_{2}\right)}+\frac{q_{1} q_{3} a c b}{\left(1-q_{1}\right)\left(1-q_{1} q_{3}\right)} \\
& +\frac{q_{2} b a c}{\left(1-q_{2}\right)\left(1-q_{1} q_{2}\right)}+\frac{q_{2} q_{3} b c a}{\left(1-q_{2}\right)\left(1-q_{2} q_{3}\right)} \\
& +\frac{q_{3} c a b}{\left(1-q_{3}\right)\left(1-q_{1} q_{3}\right)}+\frac{q_{2} q_{3}^{2} c b a}{\left(1-q_{3}\right)\left(1-q_{2} q_{3}\right)}
\end{aligned}
$$

On the other hand,

$$
\operatorname{maj}_{\mathbf{q}}(12)=\frac{1}{1-q_{1}}, \quad \operatorname{maj}_{\mathbf{q}}(21)=\frac{q_{2}}{1-q_{2}},
$$


and

$$
\begin{aligned}
H= & \frac{1}{1-q_{1} q_{2}}\left(\frac{[a,[b, c]]}{1-q_{1}}+\frac{q_{2}[b,[a, c]]}{1-q_{2}}\right) \\
= & \frac{1}{1-q_{1} q_{2}}\left(\frac{a b c-a c b-b c a+c b a}{1-q_{1}}+\frac{q_{2}(b a c-b c a-a c b+c a b)}{1-q_{2}}\right) \\
= & \frac{\left(\left(1-q_{2}\right) a b c-\left(1-q_{1} q_{2}\right) a c b+\left(q_{2}-q_{1} q_{2}\right) b a c-\left(1-q_{1} q_{2}\right) b c a+\left(q_{2}-q_{1} q_{2}\right) c a b+\left(1-q_{2}\right) c b a\right)}{\left(1-q_{1}\right)\left(1-q_{2}\right)\left(1-q_{1} q_{2}\right)} \\
= & \frac{a b c}{\left(1-q_{1}\right)\left(1-q_{1} q_{2}\right)}-\frac{a c b}{\left(1-q_{1}\right)\left(1-q_{2}\right)}+\frac{q_{2} b a c}{\left(1-q_{2}\right)\left(1-q_{1} q_{2}\right)} \\
& -\frac{b c a}{\left(1-q_{1}\right)\left(1-q_{2}\right)}+\frac{q_{2} c a b}{\left(1-q_{2}\right)\left(1-q_{1} q_{2}\right)}+\frac{c b a}{\left(1-q_{1}\right)\left(1-q_{1} q_{2}\right)} .
\end{aligned}
$$

Since $q_{1} q_{2} q_{3}=1$, this means that

$$
k_{3}(\mathbf{q})(a, b, c)=H .
$$

If $q$ is a primitive root of 1 of degree 3 and $q_{i}=q$, then $(1-q)\left(1-q^{2}\right)=1-q-$ $q^{2}+q^{3}=3$. Therefore in this case we obtain the Klyachko element

$$
k_{3}(\mathbf{q})=k_{3}
$$

These kind of calculations can be done for any $n$.

As a corollary of Theorem 1 we obtain an exact expression of $k_{n}\left(a_{1}, \ldots, a_{n}\right)$ as a Lie element.

Corollary 2 If $q^{n}=1, q^{m} \neq 1,0<m<n$, then

$$
\sum_{\sigma \in S_{n}} q^{\operatorname{maj}(\sigma)} a_{\sigma(1)} \cdots a_{\sigma(n)}=\sum_{\tau \in S_{n-1}} q^{\operatorname{maj}(\tau)}\left[a_{\tau(1)},\left[a_{\tau(2)},\left[\cdots,\left[a_{\tau(n-1)}, a_{n}\right] \cdots\right]\right]\right.
$$

Let $\amalg$ be the shuffle product on a space of words $A$. For elements $u=$ $x_{1} \ldots x_{k}, v=y_{1} \ldots y_{l} \in A$ recall that $u \sqcup v$ is defined as the sum of elements of the form $w=z_{1} \ldots z_{k+l}$ such that $\left\{z_{1}, \ldots, z_{k+l}\right\}=\left\{x_{1}, \ldots, x_{k}, y_{1}, \ldots, y_{l}\right\}$ and if $z_{i_{1}}=$ $x_{1}, \ldots, z_{i_{k}}=x_{k}, z_{j_{1}}=y_{1}, \ldots z_{j_{l}}=y_{l}$, then $i_{1}<i_{2}<\cdots<i_{k}, j_{1}<j_{2}<\cdots<j_{l}$. For $w \in A$ we say that $w$ is a part of the shuffle product $u \sqcup v$ and denote $w \in u \sqcup v$ if $w$ appears as a summand in $u \sqcup v$. For example,

$$
a b \sqcup c d=a b c d+a c b d+a c d b+c a b d+c a d b+c d a b
$$

and $a c d b$ is a part of $a b \sqcup c d$, but $a d c b$ is not.

By Lemma 5 (see below) one can reformulate the above results in terms of majorindices and shuffle products. 
Corollary 3 If $q_{1} \cdots q_{n}=1$, but $q_{i_{1}} \cdots q_{i_{r}} \neq 1$, for any proper subset $\left\{i_{1}, \ldots, i_{r}\right\} \subset$ $[n]$, then for any permutation $\sigma \in S_{n}$,

$$
\operatorname{maj}_{\mathbf{q}}(\sigma)=\frac{(-1)^{|R(\sigma)|} q_{n}}{\left(q_{n}-1\right)} \sum_{w \in L(\sigma) \amalg r e v(R(\sigma))} \operatorname{maj}_{\mathbf{q}}(w),
$$

where $L(\sigma)$ and $R(\sigma)$ are the left and right parts of $\sigma$.

Corollary 4 If $q^{n}=1, q^{m} \neq 1,0<m<n$, then for any permutation $\sigma \in S_{n}$,

$$
q^{\operatorname{maj}(\sigma)}=(-1)^{|R(\sigma)|} \sum_{w \in L(\sigma) \amalg \operatorname{rev}(R(\sigma))} q^{\operatorname{maj}(w)},
$$

where $L(\sigma)$ and $R(\sigma)$ are the left and right parts of $\sigma$.

\section{Proof of Theorem 1}

Let $S_{n, r}$ be the set of shuffle permutations,

$$
S_{n, r}=\left\{\sigma \in S_{n} \mid \sigma(1)<\cdots \sigma(r), \sigma(r+1)<\cdots<\sigma(n)\right\} .
$$

Lemma 5 For any $a_{1}, \ldots, a_{n} \in A$,

$$
\begin{aligned}
& {\left[a_{1},\left[a_{2},\left[\cdots\left[a_{n-1}, a_{n}\right] \cdots\right]\right]\right.} \\
& \quad=\sum_{r=0}^{n-1} \sum_{\sigma \in S_{n-1, r}}(-1)^{r} a_{\sigma(1)} \cdots a_{\sigma(r)} a_{n} \operatorname{rev}\left(a_{\sigma(r+1)} \cdots a_{\sigma(n-1)}\right) .
\end{aligned}
$$

Proof Follows from Theorem 8.16 [9].

Lemma 6 Let $u$ and $v$ be complementary words on $[n]$ with $|u|=k,|v|=l$. Then

$$
\sum_{w \in u \sqcup v} \operatorname{maj}_{\mathbf{q}}(w)=\frac{\left(1-q_{1} \ldots q_{n}\right)}{\left(1-q_{u(1)} \cdots q_{u(k)}\right)\left(1-q_{v(1)} \cdots q_{v(l)}\right)} \operatorname{maj}_{\mathbf{q}}(u) m a j_{\mathbf{q}}(v) .
$$

Proof Let $\langle$,$\rangle be inner (scalar) product on \mathcal{F}$ defined by $\langle u, v\rangle=\delta_{u, v}$ for any words $u$ and $v$ and extended to $\mathcal{F}$ by linearity. By the following statement (see end of Sect. 2 of the paper [7]),

$$
\begin{aligned}
& \left\langle k_{n}(\mathbf{q}), u \sqcup v\right\rangle \\
& \quad=\left(1-q_{1} \cdots q_{n}\right) \frac{\left(\prod_{j \in D(u)} q_{u(1)} \cdots q_{u(n)}\right)\left(\prod_{j \in D(v)} q_{v(1)} \cdots q_{v(n)}\right)}{\left(\prod_{i=1}^{k} q_{u(1)} \cdots q_{u(i)}\right)\left(\prod_{j=1}^{l} q_{v(1)} \cdots q_{v(j)}\right)}
\end{aligned}
$$

we have

$$
\left\langle k_{n}(\mathbf{q}), u \sqcup v\right\rangle=\frac{\left(1-q_{1} \cdots q_{n}\right) \operatorname{maj}_{\mathbf{q}}(u) \operatorname{maj}_{\mathbf{q}}(v)}{\left(1-q_{u(1)} \cdots q_{u(k)}\right)\left(1-q_{v(1)} \cdots q_{v(l)}\right)} .
$$


It remains to note that

$$
\left\langle k_{n}(\mathbf{q}), u \sqcup v\right\rangle=\sum_{w \in u \amalg v} \operatorname{maj}_{\mathbf{q}}(w) .
$$

Lemma 7 For any $\tau \in S_{n}$,

$$
\operatorname{maj}_{\mathbf{q}}(\tau)=\frac{(-1)^{|\tau|+1}\left(\prod_{j \in \operatorname{Des}(\operatorname{rev}(\tau))} q_{\tau(1)} \cdots q_{\tau(n-j)}\right)^{-1}}{\prod_{i=1}^{n-1}\left(1-\left(q_{\tau(1)} \cdots q_{\tau(i)}\right)^{-1}\right)}
$$

Proof For $\tau \in S_{n}$ let

$$
\begin{aligned}
& \operatorname{Des}(\tau)=\{i \in[n-1] \mid \tau(i)>\tau(i+1)\}, \\
& \operatorname{Rise}(\tau)=\{i \in[n-1] \mid \tau(i)<\tau(i+1)\}
\end{aligned}
$$

be sets of descent and rise indices. Then

$$
\begin{gathered}
\operatorname{Des}(\tau) \cup \operatorname{Rise}(\tau)=\{1,2, \ldots, n-1\}, \\
\operatorname{Des}(\tau) \cap \operatorname{Rise}(\tau)=\emptyset, \\
|\operatorname{Rise}(\tau)|=n-1-|\operatorname{Des}(\tau)|, \\
\operatorname{Des}(\operatorname{rev}(\tau))=\{n, \ldots, n\}-\operatorname{rev}(\operatorname{Rise}(\tau))
\end{gathered}
$$

(here $(n, \ldots, n)$ is a sequence with components $n$ and length $|\operatorname{Rise}(\tau)|)$. Thus,

$$
\operatorname{Rise}(\tau)=\{n-j \mid j \in \operatorname{Des}(\operatorname{rev}(\tau))\} .
$$

Therefore,

$$
\begin{aligned}
& \frac{(-1)^{n+1}}{\prod_{j \in \operatorname{Des}(\operatorname{rev}(\tau))} q_{\tau(1)} \cdots q_{\tau(n-j)} \prod_{i=1}^{n-1}\left(1-\left(q_{\tau(1)} \cdots q_{\tau(i)}\right)^{-1}\right)} \\
& =\frac{(-1)^{n+1} \prod_{i=1}^{n-1}\left(q_{\tau(1)} \cdots q_{\tau(i)}\right)}{\prod_{j \in \operatorname{Des}(\operatorname{rev}(\tau))} q_{\tau(1)} \cdots q_{\tau(n-j)} \prod_{i=1}^{n-1}\left(-1+\left(q_{\tau(1)} \cdots q_{\tau(i)}\right)\right)} \\
& =\frac{\prod_{i=1}^{n-1}\left(q_{\tau(1)} \cdots q_{\tau(i)}\right)}{\prod_{j \in \operatorname{Des}(\operatorname{rev}(\tau))} q_{\tau(1)} \cdots q_{\tau(n-j)} \prod_{i=1}^{n-1}\left(1-q_{\tau(1)} \cdots q_{\tau(i)}\right)} \\
& =\frac{\prod_{i \in \operatorname{Des}(\tau)} q_{\tau(1)} \cdots q_{\tau(i)}}{\prod_{i=1}^{n-1}\left(1-q_{\tau(1)} \cdots q_{\tau(i)}\right)} \\
& =m_{j_{\mathbf{q}}(\tau) .}
\end{aligned}
$$


Lemma 8 Let $u$ and $v$ be complementary nonempty words on $[n-1]$. Let $q_{1} \cdots q_{n}=$ 1 , but $q_{i_{1}} \cdots q_{i_{r}} \neq 1$, for any proper subset $\left\{i_{1}, \ldots, i_{r}\right\} \subset\{1, \ldots, n\}$. Then

$$
\operatorname{maj}_{\mathbf{q}}(u n \operatorname{rev}(v))=\frac{(-1)^{|v|} \operatorname{maj}_{\mathbf{q}}(u) \operatorname{maj}_{\mathbf{q}}(v)}{\left(1-\prod_{i=1}^{|u|} q_{u(i)}\right)\left(1-\prod_{j=1}^{|v|} q_{v(j)}\right)} .
$$

Proof Let $\sigma=u n \operatorname{rev}(v)$. Note that $\sigma^{-1}(n)=|u|+1$ and $|u| \notin \operatorname{Des}(\sigma)$. Therefore,

$$
\begin{aligned}
\operatorname{maj}_{\mathbf{q}}(\sigma) & \\
= & \frac{\prod_{j \in \operatorname{Des}(\sigma)} q_{\sigma(1)} \cdots q_{\sigma(j)}}{\prod_{i=1}^{n-1}\left(1-q_{\sigma(1)} \cdots q_{\sigma(i)}\right)} \\
= & \frac{\prod_{j \in \operatorname{Des}(\sigma), j<|u|} q_{u(1)} \cdots q_{u(j)}}{\prod_{i=1}^{|u|-1}\left(1-q_{u(1)} \cdots q_{u(i)}\right)} \\
& \times \frac{\left(q_{n} \prod_{j=1}^{|u|} q_{\sigma(j)}\right)}{\left(1-q_{u(1)} \cdots q_{u|u|)}\right)\left(1-q_{u(1)} \cdots q_{u(|u|)} q_{n}\right)} \\
& \times \frac{\prod_{j \in \operatorname{Des}(\operatorname{rev}(v))} q_{u(1)} \cdots q_{u(|u|)} q_{n} q_{v(|v|-j+1)} \cdots q_{v(|v|)}}{\prod_{i=1}^{|v|-1}\left(1-q_{u(1)} \cdots q_{u(|u|)} q_{n} q_{v(1+i)} \cdots q_{v(|v|)}\right)}
\end{aligned}
$$

Now use the condition

$$
q_{u(1)} \cdots q_{u(|u|)} q_{n} q_{v(1)} \cdots q_{v(|v|)}=q_{1} \cdots q_{n}=1 .
$$

We have

$$
\begin{aligned}
& \operatorname{maj}_{\mathbf{q}}(\sigma) \\
&=\operatorname{maj}_{\mathbf{q}}(u) \times \frac{1}{\left(1-q_{u(1)} \cdots q_{u(|u|)}\right)\left(-1+q_{v(1)} \cdots q_{v(|v|)}\right)} \\
& \quad \times \frac{\prod_{j \in \operatorname{Des}(\operatorname{rev}(v))}\left(q_{v(1)} \cdots q_{v(|v|-j)}\right)^{-1}}{\prod_{i=1}^{|v|-1}\left(1-\left(q_{v(1)} \cdots q_{v(i)}\right)^{-1}\right)} .
\end{aligned}
$$

It remains to use Lemma 7.

Proof of Theorem 1 For any $\sigma \in S_{n}$ let us calculate the coefficient of $a_{\sigma(1)} \cdots a_{\sigma(n)}$. On the left hand side of (1) it is $\operatorname{maj}_{\mathbf{q}}(\sigma)$.

Now consider the coefficient on the right hand side of (1). Denote it as $\lambda_{\sigma}$. If $r=\sigma^{-1}(n)$, then $u=L(\sigma)=\sigma(1) \cdots \sigma(r-1)$ and $v=R(\sigma)=\sigma(r+1) \cdots \sigma(n)$. Then $a_{\sigma(1)} \cdots a_{\sigma(n)}$ appears only in terms of a form

$$
\frac{\operatorname{maj}_{\mathbf{q}}(w)\left[a_{w(1)},\left[\cdots\left[a_{w(n-1)}, a_{n}\right] \cdots\right]\right]}{\left(1-q_{1} \cdots q_{n-1}\right)},
$$


where $w \in u \sqcup \operatorname{rev}(v)$, with coefficient $(-1)^{|v|}$. Hence, by Lemma 5,

$$
\lambda_{\sigma}=\sum_{w \in u \amalg r e v(v)} \frac{(-1)^{|v|}}{\left(1-q_{1} \cdots q_{n-1}\right)} \operatorname{maj}_{\mathbf{q}} w .
$$

Since $u$ and $v$ are complementary words on [n-1], by Lemma 6

$$
\lambda_{\sigma}=\frac{(-1)^{|v|}}{\left(1-q_{u(1)} \cdots q_{u(k)}\right)\left(1-q_{v(1)} \cdots q_{v(l)}\right)} \operatorname{maj}_{\mathbf{q}}(u) m a j_{\mathbf{q}}(\operatorname{rev}(v)) .
$$

By Lemma 8 we see that

$$
\lambda_{\sigma}=m a j_{\mathbf{q}}(u n v)
$$

So, coefficients at $a_{\sigma(1)} \cdots a_{\sigma(n)}$ on the left and right hand sides of (1) are equal for any $\sigma \in S_{n}$. Theorem 1 is proved.

\section{Additional remarks}

Remark 1 It will be interesting to study associative algebra as an $n$-algebra under $k_{n}$ as an $n$-ary multiplication. For example, for $n=3$ the 3 -multiplication $[a, b, c]$ defined by

$$
\left[a_{1}, a_{2}, a_{3}\right]=\sum_{\sigma \in S_{3}} q^{m a j(\sigma)} a_{\sigma(1)} a_{\sigma(2)} a_{\sigma(3)}, \quad q=-\frac{1}{2} \pm i \frac{\sqrt{3}}{2},
$$

can be re-written as

$$
[a, b, c]=(a b) c+(c b) a+q((b a) c+(c a) b)+q^{2}((a c) b+(b c) a)
$$

It satisfies the cyclic $q$-identity of 3-degree 1,

$$
[a, b, c]=q[c, a, b],
$$

and the following 25 terms identity of 3 -degree 2

$$
\begin{aligned}
& -q^{2}[a, b,[c, d, e]]-q[a, b,[d, c, e]]+[a, c,[b, d, e]]+q[a, c,[d, b, e]] \\
& -[a, d,[b, c, e]]+q^{2}[a, d,[c, b, e]]-[a, e,[b, c, d]]+q^{2}[a, e,[c, b, d]] \\
& +q^{2}[b, a,[c, d, e]]+[b, a,[d, c, e]]-q[b, c,[a, d, e]]-[b, c,[d, a, e]] \\
& +q[b, d,[a, c, e]]-q^{2}[b, d,[c, a, e]]+q[b, e,[a, c, d]]-q^{2}[b, e,[c, a, d]] \\
& -[c, a,[b, d, e]]-q^{2}[c, a,[d, b, e]]+q[c, b,[a, d, e]]+q^{2}[c, b,[d, a, e]] \\
& -q[c, d,[a, b, e]]+[c, d,[b, a, e]]-q[c, e,[a, b, d]]+[c, e,[b, a, d]] \\
& +\left(q-q^{2}\right)[d, e,[a, b, c]]=0 .
\end{aligned}
$$


540

J Algebra Comb (2011) 33: 531-542

Remark 2 The Dynkin-Specht-Wever theorem allows us to check whether an alemont $X \in \mathcal{F}$ can be presented as a linear combination of Lie commutators. Let us give some modification of this theorem that allows to write a multilinear Lie element as a linear combination of Lie base elements. The algorithm is the following:

Step 1 Write multilinear element $X$ as a linear combination of Lie commutators. One can use here the Dynkin-Specht-Wever theorem.

Step 2 Write all Lie commutators in a right-bracketed form by the rule (Jacobi identity) $[[a, b] c]:=[a,[b, c]]-[b,[a, c]]$.

Step 3 Write any right-bracketed Lie commutator $\left[a_{i_{1}},\left[\cdots\left[a_{i_{n-1}}, a_{i_{n}}\right] \cdots\right]\right.$ as a linear combination of multilinear base elements $\left[a_{\sigma(1)},\left[\cdots\left[a_{\sigma(n-1)}, a_{n}\right] \cdots\right]\right]$, where $\sigma \in S_{n-1}$. Here one can use the following formula:

$$
\begin{aligned}
{\left[a_{n},\right.} & {\left.\left[a_{1},\left[\cdots,\left[a_{n-2}, a_{n-1}\right]\right]\right]\right] } \\
= & \sum_{r=0}^{n-2} \sum_{\sigma \in S_{n-1, r+1}, \sigma(r+1)=n-1}\left[a_{\sigma(1)},\left[\cdots \left[a_{\sigma(r)},\left[a_{n-1},\left[a_{\sigma(n-2)},\right.\right.\right.\right.\right. \\
& {\left.\left.\left.\left.\left.\left[\cdots\left[a_{\sigma(r+1)}, a_{n}\right]\right]\right]\right]\right]\right]\right] . }
\end{aligned}
$$

Example Let us present an element $X=\left[\left[a_{2}, a_{5}\right]\right.$, $\left.\left[\left[a_{3}, a_{4}\right], a_{1}\right]\right]$ as a linear combingtimon of Lie base elements. We can begin from step 2,

$$
\begin{aligned}
X= & {\left[a_{2},\left[a_{5},\left[a_{3},\left[a_{4}, a_{1}\right]\right]\right]\right]-\left[a_{2},\left[a_{5},\left[a_{4},\left[a_{3}, a_{1}\right]\right]\right]\right]-\left[a_{5},\left[a_{2},\left[a_{3},\left[a_{4}, a_{1}\right]\right]\right]\right] } \\
& +\left[a_{5},\left[a_{2},\left[a_{4},\left[a_{3}, a_{1}\right]\right]\right]\right] .
\end{aligned}
$$

By (2) we have

$$
\begin{aligned}
{\left[a_{5},\left[a_{3},\left[a_{4}, a_{1}\right]\right]\right]=} & -\left[a_{3},\left[a_{4},\left[a_{1}, a_{5}\right]\right]\right]+\left[a_{3},\left[a_{1},\left[a_{4}, a_{5}\right]\right]\right] \\
& +\left[a_{4},\left[a_{1},\left[a_{3}, a_{5}\right]\right]\right]-\left[a_{1},\left[a_{4},\left[a_{3}, a_{5}\right]\right]\right],
\end{aligned}
$$

and,

$$
\begin{aligned}
{\left[a_{2},\left[a_{5},\left[a_{3},\left[a_{4}, a_{1}\right]\right]\right]\right] } \\
=-\left[a_{2},\left[a_{3},\left[a_{4},\left[a_{1}, a_{5}\right]\right]\right]\right]+\left[a_{2},\left[a_{3},\left[a_{1},\left[a_{4}, a_{5}\right]\right]\right]\right]+\left[a_{2},\left[a_{4},\left[a_{1},\left[a_{3}, a_{5}\right]\right]\right]\right] \\
\quad-\left[a_{2},\left[a_{1},\left[a_{4},\left[a_{3}, a_{5}\right]\right]\right]\right] .
\end{aligned}
$$

Similarly,

$$
\begin{aligned}
{\left[a_{2},\left[a_{5},\left[a_{4},\left[a_{3}, a_{1}\right]\right]\right]\right] } \\
=-\left[a_{2},\left[a_{4},\left[a_{3},\left[a_{1}, a_{5}\right]\right]\right]\right]+\left[a_{2},\left[a_{4},\left[a_{1},\left[a_{3}, a_{5}\right]\right]\right]\right]+\left[a_{2},\left[a_{3},\left[a_{1},\left[a_{4}, a_{5}\right]\right]\right]\right] \\
\quad-\left[a_{2},\left[a_{1},\left[a_{3},\left[a_{4}, a_{5}\right]\right]\right]\right] .
\end{aligned}
$$

By (2),

$$
\left[a_{5},\left[a_{2},\left[a_{3},\left[a_{4}, a_{1}\right]\right]\right]\right]
$$

Springer 


$$
\begin{aligned}
= & {\left[a_{1},\left[a_{4},\left[a_{3},\left[a_{2}, a_{5}\right]\right]\right]\right]-\left[a_{2},\left[a_{1},\left[a_{4},\left[a_{3}, a_{5}\right]\right]\right]\right]+\left[a_{2},\left[a_{3},\left[a_{1},\left[a_{4}, a_{5}\right]\right]\right]\right] } \\
& -\left[a_{2},\left[a_{3},\left[a_{4},\left[a_{1}, a_{5}\right]\right]\right]\right]+\left[a_{2},\left[a_{4},\left[a_{1},\left[a_{3}, a_{5}\right]\right]\right]\right] \\
& -\left[a_{3},\left[a_{1},\left[a_{4},\left[a_{2}, a_{5}\right]\right]\right]\right]+\left[a_{3},\left[a_{4},\left[a_{1},\left[a_{2}, a_{5}\right]\right]\right]\right] \\
& -\left[a_{4},\left[a_{1},\left[a_{3},\left[a_{2}, a_{5}\right]\right]\right]\right], \\
{\left[a_{5},\left[a_{2},\left[a_{4},\left[a_{3}, a_{1}\right]\right]\right]\right] } & \\
= & {\left[a_{1},\left[a_{3},\left[a_{4},\left[a_{2}, a_{5}\right]\right]\right]\right]-\left[a_{2},\left[a_{1},\left[a_{3},\left[a_{4}, a_{5}\right]\right]\right]\right]+\left[a_{2},\left[a_{3},\left[a_{1},\left[a_{4}, a_{5}\right]\right]\right]\right] } \\
& +\left[a_{2},\left[a_{4},\left[a_{1},\left[a_{3}, a_{5}\right]\right]\right]\right]-\left[a_{2},\left[a_{4},\left[a_{3},\left[a_{1}, a_{5}\right]\right]\right]\right] \\
& -\left[a_{3},\left[a_{1},\left[a_{4},\left[a_{2}, a_{5}\right]\right]\right]\right]-\left[a_{4},\left[a_{1},\left[a_{3},\left[a_{2}, a_{5}\right]\right]\right]\right] \\
& +\left[a_{4},\left[a_{3},\left[a_{1},\left[a_{2}, a_{5}\right]\right]\right]\right] .
\end{aligned}
$$

Therefore,

$$
\begin{aligned}
X= & {\left[a_{1},\left[a_{3},\left[a_{4},\left[a_{2}, a_{5}\right]\right]\right]\right]-\left[a_{1},\left[a_{4},\left[a_{3},\left[a_{2}, a_{5}\right]\right]\right]\right]-\left[a_{3},\left[a_{4},\left[a_{1},\left[a_{2}, a_{5}\right]\right]\right]\right] } \\
& +\left[a_{4},\left[a_{3},\left[a_{1},\left[a_{2}, a_{5}\right]\right]\right]\right] .
\end{aligned}
$$

Remark 3 Klyachko element has one more generalization. In [6] a Lie idempotent was constructed that generalises three other well-known idempotents. This generalization is the $q$-Solomon idempotent

$$
\phi_{n}(q)=\frac{1}{n} \sum_{\sigma \in S_{n}} \frac{(-1)^{\operatorname{des}(\sigma)} q^{\operatorname{maj}(\sigma)-\left(\begin{array}{c}
d(\sigma)+1 \\
2
\end{array}\right)}}{\left[\begin{array}{l}
n-1 \\
d(\sigma)
\end{array}\right]_{q}} \sigma .
$$

It has the following properties:

$$
\phi_{n}(\omega)=k_{n}(\omega),
$$

is the Klyachko element if $\omega$ is a primitive root of degree $n$,

$$
\phi_{n}(0)=[\cdots[1,2], \ldots, n]
$$

is the Dynkin idempotent in case of $q=0$ and

$$
\phi_{n}(1)=\sum_{\sigma \in S_{n}} \frac{(-1)^{\operatorname{des}(\sigma)}}{\left(\begin{array}{c}
n-1 \\
\operatorname{des}(\sigma)
\end{array}\right)} \sigma
$$

gives us the (first) Euler idempotent if $q=1$. Here $\left[\begin{array}{c}n-1 \\ p\end{array}\right]_{q}$ denotes a $q$-binomial coefficient. 
One can establish that the $q$-Solomon idempotent has a similar Lie presentation by base elements,

$$
\phi_{n}(q)=\frac{1}{n} \sum_{\sigma \in S_{n}, \sigma(n)=n} \frac{(-1)^{\operatorname{des}(\sigma)} q^{\operatorname{maj}(\sigma)-\left(\begin{array}{c}
d(\sigma)+1 \\
2
\end{array}\right)}}{\left[\begin{array}{l}
n-1 \\
d(\sigma)
\end{array}\right]_{q}}[\sigma]
$$

where $[\sigma]$ denotes the Lie commutator $[\sigma(1),[\cdots[\sigma(n-1), n] \cdots]]$. It follows from the following property of major indices

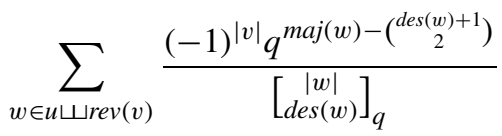

$$
\begin{aligned}
& =-\frac{(-1)^{\operatorname{des}(u)+\operatorname{des}(v)} q^{\operatorname{maj}(u)+\operatorname{maj}(v)+(|u|+1)(\operatorname{des}(v)+1)-\left(\begin{array}{c}
\operatorname{des}(u)+\operatorname{des}(v)+2 \\
2
\end{array}\right)}}{\left[\begin{array}{c}
|u|+|v| \\
\operatorname{des}(u)+\operatorname{des}(v)+1
\end{array}\right]_{q}} .
\end{aligned}
$$

In particular, the Lie presentation for Dynkin element is given by

$$
\begin{aligned}
& {\left[\left[\cdots\left[a_{1}, a_{2}\right], \cdots\right], a_{n}\right]} \\
& =\sum_{\sigma \in S_{n}, \operatorname{maj}(\sigma)=\left(\begin{array}{c}
\operatorname{des}(\sigma)+1 \\
2
\end{array}\right), \sigma(n)=n}(-1)^{\operatorname{des}(\sigma)}\left[a_{\sigma(1)},\left[\cdots\left[a_{\sigma(n-1)}, a_{n}\right] \cdots\right]\right]
\end{aligned}
$$

and the Euler element has the following Lie presentation:

$$
\sum_{\sigma \in S_{n}} \frac{(-1)^{\operatorname{des}(\sigma)}}{\left(\begin{array}{c}
n-1 \\
\operatorname{des}(\sigma)
\end{array}\right)} a_{\sigma(1)} \cdots a_{\sigma(n)}=\sum_{\sigma \in S_{n}, \sigma(n)=n} \frac{(-1)^{\operatorname{des}(\sigma)}}{\left(\begin{array}{c}
n-1 \\
\operatorname{des}(\sigma)
\end{array}\right)}\left[a_{\sigma(1)},\left[\cdots\left[a_{\sigma(n-1)}, a_{n}\right] \cdots\right]\right] .
$$

Acknowledgements I am grateful to the anonymous referee for careful reading of the text and essential remarks.

\section{References}

1. Bergeron, F., Bergeron, N., Garsia, A.M.: Idempotents for the free Lie algebra and $q$-enumeration. In: Stanton, D. (ed.) Invariant Theory and Tableaux. IMA Vol. Math. Appl., vol. 19, pp. 166-190. Springer, New York (1990)

2. Blessenohl, D., Laue, H.: A basis construction for free Lie algebras. Expo. Math. 11, 145-152 (1993)

3. Garsia, A.M.: Combinatorics of the free Lie algebra and the symmetric group. In: Analis, et cetera, pp. 309-382. Academic Press, Boston (1990)

4. Jacobson, N.: Lie Algebras. Interscience, New York (1962)

5. Klyachko, A.A.: Lie elements in a tensor algebra. Sib. Mat. Zh. 15(6), 1296-1304 (1974)

6. Krob, D., Leclerc, B., Thibon, J.-Y.: Noncommutative symmetric functions II: Transformations of alphabets. Int. J. Algebra Comput. 7, 181-264 (1997)

7. McNamara, P., Reutenauer, C.: P-partitions and multi-parameter Klyachko idempotent. Electron. J. Comb. 11(2), \#R21 (2005)

8. Patras, F., Reutenauer, C., Schocker, M.: On the Garsia Lie idempotent. Can. Math. Bull. 48(3), 445454 (2005)

9. Reutenauer, C.: Free Lie Algebras. Clarendon Press, Oxford (1993) 